\title{
Minireview
}

\section{Editing plants for virus resistance using CRISPR-Cas}

\author{
J. C. GREEN, J. S. HU*
}

Department of Plant and Environmental Protection Sciences, University of Hawaii, 3190 Maile Way, Honolulu, HI 96822, USA

Received September 30, 2016; revised October 24, 2016; accepted March 10, 2017

\begin{abstract}
Summary. - This minireview summarizes recent advancements using the clustered regularly interspaced palindromic repeats-associated nuclease systems (CRISPR-Cas) derived from prokaryotes to breed plants resistant to DNA and RNA viruses. The CRISPR-Cas system represents a powerful tool able to edit and insert novel traits into plants precisely at chosen loci offering enormous advantages to classical breeding. Approaches to engineering plant virus resistance in both transgenic and non-transgenic plants are discussed. Iterations of the CRISPR-Cas system, FnCas9 and C2c2 capable of editing RNA in eukaryotic cells offer a particular advantage for providing resistance to RNA viruses which represent the great majority of known plant viruses. Scientists have obtained conflicting results using gene silencing technology to produce transgenic plants resistant to geminiviruses. CRISPR-Cas systems engineered in plants to target geminiviruses have consistently reduced virus accumulation providing increased resistance to virus infection. CRISPR-Cas may provide novel and reliable approaches to control geminiviruses and other ssDNA viruses such as Banana bunchy top virus (BBTV).
\end{abstract}

Keywords: CRISPR-Cas; virus resistance; plant virus

\section{Contents:}

1. Introduction

2. CRISPR-Cas-mediated resistance for DNA viruses

3. Possible CRISPR-Cas-mediated resistance for RNA viruses

4. CRISPR-Cas-introduced host mutations confer RNA virus resistance

5. Non-random transformation for production of transgenic plants

"Corresponding author. E-mail: johnhu@hawaii.edu; phone: +808956-7281.

Abbreviations: $\mathrm{BBTV}=$ Banana bunchy top virus; $\mathrm{BSCTV}=$ Beet severe curly top virus; $\mathrm{C} 2 \mathrm{c} 2$ = class 2 type VI-A CRISPR-Cas effector; CRISPR-Cas $=$ clustered regularly interspaced palindromic repeatsassociated nuclease systems; crRNA = CRISPR RNA; DSB = double strand breaks; FnCas9 = Cas endonuclease derived from Franciscella novicida; $\mathrm{HDR}=$ homology-directed repair; NHEJ = non-homologous end-joining; sgRNA = small guide RNA; tracRNA = transactivating RNA; TYLCV = Tomato yellow leaf curl virus
6. Plant tissue culture-independent genome editing using viruses

7. Conclusion and future perspectives

\section{Introduction}

Recent advances in genome editing technologies using sequence-specific nucleases (SSNs) are revolutionizing the life sciences, especially clustered regularly interspaced palindromic repeats-associated nuclease systems (CRISPRCas). CRISPR-Cas is a naturally occurring autoimmune system in prokaryotes that confers resistance to foreign nucleic acids (Makarova et al., 2006). The CRISPR-Cas approach to genome editing more easily allows engineering of specific targetable nuclease systems in stark contrast to other alternative SSNs such as DNA-protein recognition zinc finger nucleases (ZFNs) (Kim et al., 1996) and transcription activator-like effector nucleases (TALENs) (Christian et al., 2010). ZFNs and TALENs have not been adopted extensively 
by the plant science community, perhaps due to the burdensome requirement for two different DNA binding proteins, both with a C-terminal FokI nuclease module, to flank the targeted sequence (Belhaj et al., 2013). CRISPR-Cas genome editing offers novel alternatives to classical plant breeding and transgenic methods of crop improvement to address the ever-looming Malthusian check.

CRISPR-Cas systems in prokaryotes incorporate short, foreign transcripts from invading nucleic acids into a CRISPR array in the host genome (Bhaya et al., 2011; Wiedenheft et al., 2012). CRISPR-Cas systems I, II and III, use separate molecular mechanisms to recognize and initiate cleavage of target nucleic acids (Makarova et al., $2011 \mathrm{a}, \mathrm{b})$. In type II systems, transcripts of invading nucleic acids from the CRISPR array later transcribed as CRISPRRNA (crRNA) are post-transcriptionally processed into a complex with trans-activating RNA (tracRNA) by the widely conserved endogenous RNase III (Deltcheva et al., 2011). The protospacer adjacent motif (PAM) is a short sequence motif adjacent to the targeted sequence necessary for target recognition and cleavage in type I and II systems (Shah et al., 2013).

The type II system isolated from Streptococcus pyogenes and widely referred to as CRISPR-Cas9 is the most commonly used CRISPR-Cas system in genome editing today due to the ease of modifying the targeted site by changing a short sequence of approximately 20 nucleotides (nt) (Doudna and Charpentier, 2014). The construction of a synthetic "linker loop" or scaffold (Fig. 1a) that fuses the crRNA and tracRNA into one small guide RNA (sgRNA) has greatly facilitated design and ease of construction of multiple sgRNA iterations (Jinek et al., 2012). CRISPR-Cas systems exploit widely conserved double strand breaks (DSB) repair pathways to induce desirable changes at specific chosen loci. CRISPR-Cas systems may be engineered to take advantage of the non-homologous end-joining (NHEJ) pathway to affect the error-prone repair of DSB often resulting in frameshift mutations. Additionally CRISPR-Cas systems may be engineered to achieve the homology-directed repair (HDR) of a DNA template on an exogenous donor vector effectively allowing the insertion of foreign genes or DNA sequences (Steinert et al., 2016).

\section{CRISPR-Cas-mediated resistance for DNA viruses}

The use of various methods of transformation to produce virus-resistant plants has been well characterized in the literature. Engineering virus resistance in plants began by demonstrating a delay in disease development in transgenic tobacco plants expressing the coat protein (CP) gene from Tobacco mosaic virus (TMV) (Abel et al., 1986). Later, collaboration between the University of Hawaii and

\section{(a) CRISPR-Cas System}

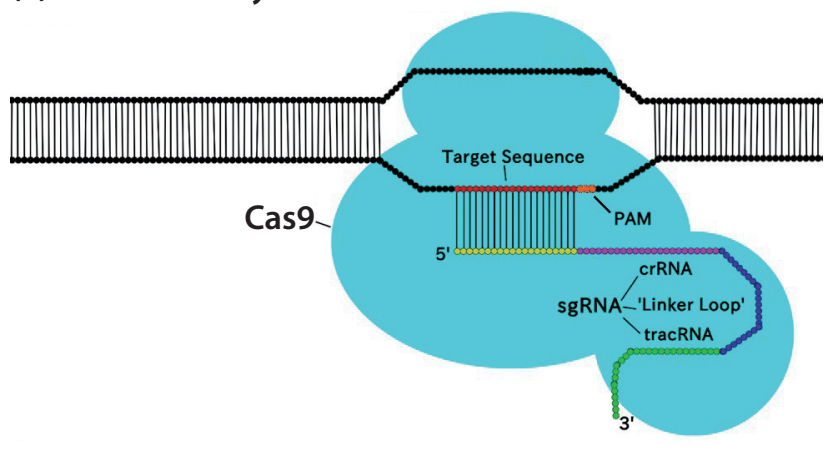

(b) Double Strand Break Repair Pathways
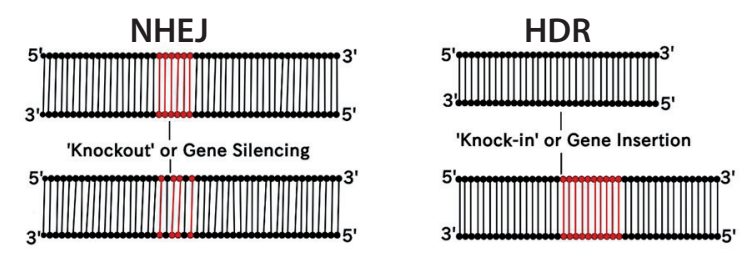

Fig. 1

Schematic representation of the two-component CRISPR-Cas system and double strand break DNA repair pathways exploited by CRISPR-Cas

(a) Cas nuclease (light blue) binding to dsDNA. The Cas nuclease is paired in the complex with a small guide RNA (sgRNA) composed of a 20-nucleotide sequence (yellow) sharing homology to the target region (red), and the crRNA (purple) fused to the tracRNA (green) by a synthetic 'linker loop' (dark blue). The sgRNA directs the Cas nuclease to the target region initiating DSB approximately $3 \mathrm{nt}$ downstream from the protospacer adjacent motif (PAM, orange). (b) The CRISPR-Cas system exploits the widely conserved double strand break DNA repair pathways to promote gene editing. NHEJ frequently induces insertions or deletions (InDels). These InDels can cause frameshift mutations that result in the knockout or silencing of gene expression. The HDR pathway can also be used for 'knock-in' or targeted gene insertion.

Cornell University resulted in a commercially marketable virus-resistant transgenic papaya (Carica papaya 'Rainbow') expressing the $\mathrm{CP}$ gene of Papaya ringspot virus (PRSV). The success of the transgenic 'Rainbow' cultivar kept Hawaii's papaya industry from collapsing under the heavy pressure of papaya ringspot disease (Gonsalves, 1998).

The CRISPR-Cas system's ability to seek out and destroy foreign nucleic acids offers an ideal mechanism to produce transgenic virus resistance in plants. Several iterations of the prokaryotic CRISPR-Cas system have been engineered in plants to confer resistance to crop diseases caused by geminiviruses in the model organisms Arabidopsis thaliana and Nicotiana benthamiana. Geminiviruses harbor circular, single-stranded DNA (ssDNA) genomes that generate double-stranded DNA (dsDNA) intermediates through rolling-circle replication (Hanley-Bowdoin et al., 2013). 
A CRISPR-Cas immune system engineered to target Bean yellow dwarf virus (BeYDV), reduced the virus load and symptoms in transgenic $N$. benthamiana by creating mutations in the BeYDV genome and reducing copy numbers (Baltes et al., 2015). A CRISPR-Cas immune system engineered to target Beet severe curly top virus (BSCTV) in transgenic $N$. benthamiana inhibited virus accumulation during transient assays and introduced mutations through the NHEJ repair pathway (Ji et al., 2015). Further, transgenic N. benthamiana and $A$. thaliana plants overexpressing the same CRISPR-Cas system were highly resistant to BSCTV infection. BSCTV accumulation in these plants was inhibited and efficiency of reducing virus accumulation was correlated with the expression level of Cas9 (Ji et al., 2015). A CRISPR-Cas immune system engineered to target Tomato yellow leaf curl virus (TYLCV) in transgenic N. benthamiana delayed or reduced accumulation of TYLCV DNA and abolished or significantly reduced symptoms (Ali et al., 2015a). Further, by targeting the highly conserved stem-loop sequence of the origin of replication (ORI), a single sgRNA was able to confer resistance to multiple viruses in transgenic $N$. benthamiana . Targeting the stem-loop sequence of the ORI in the intragenic region (IR) of TYLCV conferred resistance to the geminiviruses TYLCV, BSCTV and merremia mosaic virus (MeMV) (Ali et al., 2015a).

Geminiviruses appear to exhibit altered pathogenicity and symptom severity due to a recombination or reassortment of viral genomes (Hanley-Bowdoin et al., 2013). Investigation into CRISPR-generated virus mutants found that CRISPRCas systems engineered to target the coding sequences of geminiviruses resulted in the generation of viral variants capable of replication and systemic movement (Ali et al., 2016). Targeting of the non-coding IR region of geminiviruses, however, resulted in the interference of replication and eliminated viral variants (Ali et al., 2016).

Previously available plant transformation technologies have failed to offer a solution to the agriculturally important ssDNA viruses such as BBTV despite numerous attempts (Becker et al., 2000; Borth et al., 2011; Krishna et al., 2013; Shekhawat et al., 2012). The initial success of transgenic plants engineered with CRISPR-Cas systems to combat geminiviruses points to the potential of a similar system against other ssDNA viruses, like BBTV.

\section{Possible CRISPR-Cas-mediated resistance for RNA viruses}

Although CRISPR-Cas has been successfully adapted as an immune system in plants for ssDNA geminiviruses, iterations of the system targeting plant viruses with RNA genomes, which include most plant viruses, have yet to coalesce. A CRISPR-Cas system has been engineered to target RNA in mammalian cells using a Cas endonuclease derived from Franciscella novicida (FnCas9) (Price et al., 2015). The FnCas9 CRISPR-Cas system uses a RNA-targeting guide RNA (rgRNA) for targeting endogenous mRNA in host cells. A plant codon-optimized version of FnCas9 paired with rgRNA targeting ssRNA viruses could be employed in plantae to confer resistance to important plant viruses. Recently, another RNA-targeting CRISPR-Cas system has been characterized as the Class 2 type VI-A CRISPR-Cas effector (C2c2) (Abudayyeh et al., 2016). C2c2, from the bacterium Leptotrichia shahii, can be programmed to cleave ssRNA targets carrying complementary protospacers and in bacteria can be programmed to knock down specific mRNAs (Abudayyeh et al., 2016). The C2c2 and FnCas9 CRISPRCas systems offer plant virologists yet another CRISPR-Cas systems to use against plant-pathogenic RNA viruses.

\section{CRISPR-Cas-introduced host mutations confer RNA virus resistance}

A CRISPR-Cas system was engineered to introduce targeted mutations in the recessive eukaryotic translation initiation factor eIF4E gene in cucumber (Cucumis sativus). The engineered CRISPR-Cas system exhibited broad virus immunity or resistance in T3 homozygotic mutant plants (non-transgenic, genome edited) compared to the susceptible heterozygous T3 plants (Chandrasekaran et al., 2016). Immunity to virus infection by the ssRNA ipomovirus cucumber vein yellowing virus (CVYV) and resistance to ssRNA Zucchini yellow mosaic virus (ZYMV) and Papaya ringspot virus- $W$ (PRSV-W) were also observed (Chandrasekaran et al., 2016). Further, in A. thaliana a CRISPRCas system introduced targeted mutations into eIF(iso)4E, an isoform of eIF4E, conferring complete resistance to the Potyvirus turnip mosaic virus (TuMV) (Pyott et al., 2016).

\section{Non-random transformation for production of transgenic plants}

The randomness of Agrobacterium-mediated and particle bombardment transformation methods has severely limited transgenic approaches towards conferring virus resistance in plants. Successful non-random or targeted gene insertion was first achieved in protoplast cultures of $N$. benthamiana (Li et al., 2013) and more recently by particle bombardment in maize (Zea maydis) (Svitashev et al., 2015). A CRISPR-Cas system introduced DSB of the endogenous host MIR gene in A. thaliana enabled the incorporation by HDR of a donor DNA template containing an enhanced green fluorescent protein (eGFP). The donor DNA template consisted of two homologous arms, a left arm of $733 \mathrm{bp}$ and a right arm of $825 \mathrm{bp}$, flanking the eGFP into the cleaved MIR gene locus (Zhao et al., 2016). 


\section{Plant tissue culture-independent genome editing using viruses}

Plant transformation has previously been tied to plant tissue culture due to the necessity of regenerating successful transformants through micropropagation. Plant tissue culture is time-and resource-consuming and limits the potential of this otherwise extremely useful technology. Recently, viral vectors have been used to act as delivery vehicles for the sgRNA component of the CRISPR-Cas system in transgenic $N$. benthamiana constitutively expressing Cas9. Multiple viral vectors have been used to transport CRISPRCas reagents, including the ssRNA tobravirus, Tobacco rattle virus (TRV) (Ali et al., 2015b) and the ssDNA geminivirus, Cabbage leaf curl virus (CaLCuV) (Yin et al., 2015). Donor copies of sgRNA are introduced by viral vectors at a higher frequency than traditional sgRNA delivery methods due to continued transcription by multiple copies of the replicating virus. Viral vectors disseminate the sgRNA throughout the plant, notably to the germline cells in meristematic tissues, improving the recovery of edited plants through seeds (Ali et al., 2015b).

\section{Conclusion and future perspectives}

Using CRISPR-Cas technology to confer resistance to both DNA and RNA viruses is now available for transgenic and non-transgenic plants. Site-specific insertion of transgenic DNA through CRISPR-Cas systems engineered to exploit the HDR pathway offers advantages over the random insertion methods commonly used in plants (Agrobacterium-mediated transformation and microprojectile bombardment). The CRISPR-Cas system can be engineered to insert transgenes at chosen loci, avoid unwanted positional effects and prevent the disruption of native gene function. The widespread dissemination and use of CRISPR-Cas technology to address diverse agricultural problems is a key indicator of its future potential. This system can now combat geminiviruses and may potentially be used against agriculturally important ssDNA viruses such as BBTV.

Acknowledgements. This work was supported in part by the USDA National Institute of Food and Agriculture, Hatch project HAA9025-H, managed by the College of Tropical Agriculture and Human Resources, University of Hawaii at Manoa, Honolulu, HI, USA.

\section{References}

Abel PP, Nelson RS, De B, Hoffmann N, Rogers SG, Fraley RT, Beachy RN (1986): Delay of Disease Development in
Transgenic Plants that Express the Tobacco Mosaic Virus Coat Protein Gene. Science 232, 738-743. https://doi. org/10.1126/science. 3457472

Abudayyeh OO, Gootenberg JS, Konermann S, Joung J, Slaymaker IM, Cox DBT, Shmakov S, Makarova KS, Semenova E, Minakhin L, Severinov K, Regev A, Lander ES, Koonin EV, Zhang F (2016): C2c2 is a single-component programmable RNA-guided RNA-targeting CRISPR effector. Science 353, 6299. https://doi.org/10.1126/science.aaf5573

Ali Z, Abul-faraj A, Idris A, Ali S, Tashkandi M, Mahfouz MM (2015a): CRISPR/Cas9-mediated viral interference in plants. Genome Biol. 16, 1-11. https://doi.org/10.1186/ s13059-015-0799-6

Ali Z, Abul-faraj A, Li L, Ghosh N, Piatek M, Mahjoub A, Aouida M, Piatek A, Baltes NJ, Voytas DF, Dinesh-Kumar S, Mahfouz MM (2015b): Efficient Virus-Mediated Genome Editing in Plants Using the CRISPR/Cas9 System. Mol. Plant 8, 1288-12891. https://doi.org/10.1016/j. molp.2015.02.011

Ali Z, Ali S, Tashkandi M, Zaidi SS, Mahfouz MM (2016): CRISPR/ Cas9-Mediated Immunity to Geminiviruses: Differential Interference and Evasion. Sci. Rep. 6, 26912. https://doi. org/10.1038/srep26912

Baltes NJ, Hummel AW, Konecna E, Cegan R, Bruns AN, Bisaro DM, Voytas DF (2015): Conferring resistance to geminiviruses with the CRISPR-Cas prokaryotic immune system. Nature Plants 1, 15145. https://doi.org/10.1038/ nplants.2015.145

Becker DK, Dugdale B, Smith MK, Harding RM, Dale JL (2000): Genetic transformation of Cavendish banana (Musa spp. AAA group) cv 'Grand Nain' via microprojectile bombardment. Plant Cell Reports19, 229-234. https:// doi.org/10.1007/s002990050004

Belhaj K, Chaparro-Garcia A, Kamoun S, Nekrasov V (2013): Plant genome editing made easy: targeted mutagenesis in model and crop plants using the CRISPR/Cas system. Plant Methods 9, 1-10. https://doi.org/10.1186/17464811-9-39

Bhaya D, Davison M, Barrangou R (2011): CRISPR-Cas systems in bacteria and archaea: versatile small RNAs for adaptive defense and regulation. Annu. Rev. Genet. 45, 273-297. https://doi.org/10.1146/annurev-genet-110410-132430

Borth W, Perez E, Cheah K, Chen Y, Xie WS, Gaskill D, Khalil S, Sether D, Melzer M, Wang M, Manshardt R, Gonsalves D, $\mathrm{Hu}$ JS (2011): Transgenic Banana Plants Resistant to Banana Bunchy Top Virus Infection. Acta Hortic. 897, 449457. https://doi.org/10.17660/ActaHortic.2011.897.61

Chandrasekaran J, Brumin M, Wolf D, Leibman D, Klap C, Pearlsman M, Sherman A, Arazi T, Gal-On A (2016): Development of broad virus resistance in non-transgenic cucumber using CRISPR/Cas9 technology. Mol. Plant Pathol. 17, 1140-1153. https://doi.org/10.1111/mpp.12375

Christian M, Cermak T, Doyle EL, Schmidt C, Zhang F, Hummel A, Bogdanove AJ, Voytas DF (2010): Targeting DNA doublestrand breaks with TAL effector nucleases. Genetics 186, 757-761. https://doi.org/10.1534/genetics.110.120717

Deltcheva E, Chylinski K, Sharma CM, Gonzales K, Chao Y, Pirzada ZA, Eckert MR, Vogel J, Charpentier E (2011): 
CRISPR RNA maturation by trans-encoded small RNA and host factor RNase III. Nature 471, 602-607. https:// doi.org/10.1038/nature09886

Doudna JA, Charpentier E (2014): Genome editing. The new frontier of genome engineering with CRISPR-Cas9. Science 346, 1258096. https://doi.org/10.1126/science.1258096

Gonsalves D (1998): Control Of Papaya Ringspot Virus In Papaya: A Case Study. Annu. Rev. Phytopathol. 36, 415-437. https://doi.org/10.1146/annurev.phyto.36.1.415

Hanley-Bowdoin L, Bejarano ER, Robertson D, Mansoor S (2013): Geminiviruses: masters at redirecting and reprogramming plant processes. Nat. Rev. Microbiol. 11, 777-788. https://doi.org/10.1038/nrmicro3117

Ji X, Zhang H, Zhang Y, Wang Y, Gao C (2015): Establishing a CRISPR-Cas-like immune system conferring DNA virus resistance in plants. Nature Plants 1, 15144. https://doi. org/10.1038/nplants.2015.144

Jinek M, Chylinski K, Fonfara I, Hauer M, Doudna JA, Charpentier E (2012): A Programmable Dual-RNA-Guided DNA Endonuclease in Adaptive Bacterial Immunity. Science 337, 816-821. https://doi.org/10.1126/science.1225829

Kim Y-G, Cha J, Chandrasegaran S (1996): Hybrid restriction enzymes: Zinc finger fusions to Fok I cleavage domain. PNAS 93, 1156-1160. https://doi.org/10.1073/ pnas.93.3.1156

Krishna B, Kadu AA, Vyavhare SN, Chaudhary RS, Joshi SS, Patil AB, Subramaniam VR, Sane PV (2013): RNAi-mediated resistance against banana bunchy top virus (BBTV) in'Grand Nain'banana. Acta Hortic. 974, 157-164. https:// doi.org/10.17660/ActaHortic.2013.974.18

Li JF, Norville JE, Aach J, McCormack M, Zhang D, Bush J, Church GM, Sheen J (2013): Multiplex and homologous recombination-mediated genome editing in Arabidopsis and Nicotiana benthamiana using guide RNA and Cas9. Nat. Biotechnol. 31, 688-691. https://doi.org/10.1038/ nbt.2654

Makarova KS, Grishin NV, Shabalina SA, Wolf YI, Koonin EV (2006): A putative RNA-interference-based immune system in prokaryotes: computational analysis of the predicted enzymatic machinery, functional analogies with eukaryotic RNAi, and hypothetical mechanisms of action. Biol. Direct. 1, 7. https://doi.org/10.1186/17456150-1-7

Makarova KS, Aravind L, Wolf YI, Koonin EV (2011a): Unification of Cas protein families and a simple scenario for the origin and evolution of CRISPR-Cas systems. Biol. Direct. 6, 1-27. https://doi.org/10.1186/1745-6150-6-38

Makarova KS, Haft DH, Barrangou R, Brouns SJ, Charpentier E, Horvath P, Moineau S, Mojica FJ, Wolf YI, Yakunin AF, van der Oost J, Koonin EV (2011b): Evolution and classification of the CRISPR-Cas systems. Nat. Rev. Microbiol. 9, 467-477. https://doi.org/10.1038/nrmicro2577

Price AA, Sampson TR, Ratner HK, Grakoui A, Weiss DS (2015): Cas9-mediated targeting of viral RNA in eukaryotic cells. PNAS 112, 6164-6169. https://doi.org/10.1073/ pnas. 1422340112

Pyott DE, Sheehan E, Molnar A (2016): Engineering of CRISPR/ Cas9-mediated potyvirus resistance in transgene-free Arabidopsis plants. Mol. Plant Pathol. 17, 1276-1288. https://doi.org/10.1111/mpp.12417

Shah SA, Erdmann S, Mojica FJ, Garrett RA (2013): Protospacer recognition motifs: mixed identities and functional diversity. RNA Biol. 10, 891-899. https://doi.org/10.4161/rna.23764

Shekhawat UK, Ganapathi TR, Hadapad AB (2012): Transgenic banana plants expressing small interfering RNAs targeted against viral replication initiation gene display highlevel resistance to banana bunchy top virus infection. J. Gen. Virol. 93, 1804-1813. https://doi.org/10.1099/ vir.0.041871-0

Steinert J, Schiml S, Puchta H (2016): Homology-based doublestrand break-induced genome engineering in plants. Plant Cell. Rep. 35, 1429-1438. https://doi.org/10.1007/ s00299-016-1981-3

Svitashev S, Young JK, Schwartz C, Gao H, Falco SC, Cigan AM (2015): Targeted Mutagenesis, Precise Gene Editing, and Site-Specific Gene Insertion in Maize Using Cas9 and Guide RNA. Plant Physiol. 169, 931-945. https://doi. org/10.1104/pp.15.00793

Wiedenheft B, Sternberg SH, Doudna JA (2012): RNA-guided genetic silencing systems in bacteria and archaea. Nature 482, 331-338. https://doi.org/10.1038/nature10886

Yin K, Han T, Liu G, Chen T, Wang Y, Yu AY, Liu Y (2015): A geminivirus-based guide RNA delivery system for CRISPR/ Cas9 mediated plant genome editing. Sci. Rep. 5, 14926. https://doi.org/10.1038/srep14926

Zhao Y, Zhang C, Liu W, Gao W, Liu C, Song G, Li WX, Mao L, Chen B, Xu Y, Li X, Xie C (2016): An alternative strategy for targeted gene replacement in plants using a dual-sgRNA/ Cas9 design. Sci. Rep. 6, 23890. https://doi.org/10.1038/ $\underline{\text { srep23890 }}$ 